\title{
Strong Detonation of a Certain Type of no Fragment Design and Simulation Research
}

\author{
Yongzhong $\mathrm{Ma}$ \\ Engineering University of Armed Police Force, Xi'an 710086, China \\ 754990983@qq.com
}

\begin{abstract}
Keywords: Double no fragment; Strong detonation play; The simulation; Structural reliability; Orifice overpressure
\end{abstract}

\begin{abstract}
In order to solve the problem caused by strong detonation play fragment damage, the design of the double-layer structure without fragment strong detonation, using ls-dyna nonlinear analysis tools of the strong detonation is set up simulation model of the dispersion of inner shell fragment, and shell inner shell fragment, as well as the overpressure of the spray hole are analyzed, and is verified by structural reliability test, the results show that the double-layer structure without fragment fragment eliminates the damage completely, and achieves the design requirements. This method has a certain reference significance on design and development of strong detonation.
\end{abstract}

\section{Introduction}

Blast bomb [1] is a kind of sound and light bomb, using strong sound and blinding flash generated by explosion to stimulate ears and eyes of exciting living target, it is a non-lethal police ammunition which makes targets lose the ability of acting temporarily. Its development in China starts late, its structure and materials are relatively simple, the main use is ABS alloy. When using, the damage fragments produced by ABS alloy may cause casualties on innocent people. Therefore, developing a new sound and light bomb without fragments makes sense.

Bomb blast research rely mainly on test in domestic currently, but the test lacks theoretical research, higher research costs, long development cycles and so on. But we optimize the parameters of the bomb blast by means of simulation and verify by experiment to save cost and time, and master a large bang theory data. Therefore, this article mainly studies simulation parameters on bomb blast, and verifies the feasibility of the structure without breaking sheet, laies the foundation for further optimization parameters on bomb detonation.

LS-DYNA is based on explicit nonlinear dynamic, supplemented by implicit which is general nonlinear dynamic finite element program, especially suitable for solving nonlinear structure of the high-speed collision, the impact of explosions and metal forming and other problems, at the same time can solve heat transfer, fluids and fluid-structure interaction [2]. Therefore, this article selectes LS-DYNA simulation software.

\section{Preliminary Program}

Through analysis, we think out three solutions to solve destruction fragmentation: the first one has fragments without destruction, such as: paper form of fragments; the second is completely broken fragments, such as: a material is stable performance before the explosion, completely melted or broken when explosing, and do not form anti-personnel fragmentation; the third is no fragments, such as: internal explosion and plus the opening on the housing structure to ensure that fragments remain in the internal steel. Taking the first option into account, the paper fragments may not be strong enough, detonation pressure which is not enough causes tactical and technical index reach. Considering the second solution, these materials may be hard to find or expensive. Therefore, this article chooses the third program. In order to verify the feasibility, this article preliminary designs a scheme which uses Pro / E to draw a blueprint, shown in Fig. 1. 
This ammunition uses double cavity structure. The inner shell uses ABS alloy material and the outer shell uses aluminum alloy material. Orifice diameter is $4 \mathrm{~mm}$, the angle is 45 degree and the number is 10. Taking the cost and processing technology into account, upper, middle and lower part use threaded connection, this article uses flash agent which can release energy peaked $4.1 \times 106 \mathrm{~kJ}$ though explosion, reaching the requirements of sound and light effects.

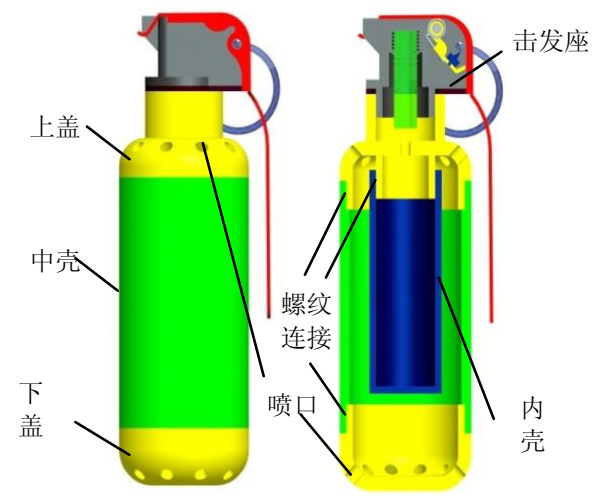

Figure 1. Preliminary program figure of Proe Model explanation:

\section{Simulation Model Establishment Based on LS-DYNA}

Simplified Model. The influence between firing mechanism of model and explosion is negligible, the explosion initiation point is located on the inner tube along the center. The ammunition is axisymmetric model, so this article sets up quarter model on simulation explosion [3] to reduce the amount of calculation, and establishes air model outside, so the final model is shown in Fig. 2.

Division of the Grid. For the rule body, this article uses the mapping hexahedral meshes, the irregularities use smart sweeping grid, the effect shows in Fig. 3.

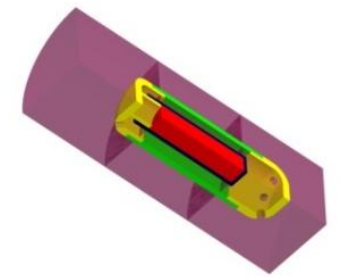

Figure 2. Air model of outsourcing

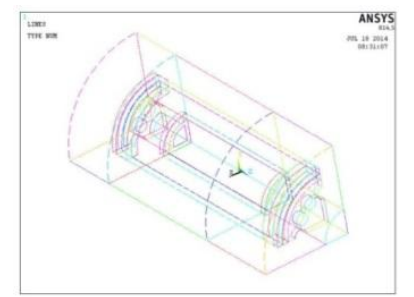

Figure 3. Meshing effect

Solving Settings. After meshing, you need to take the following settings:

(1) Generating part.

(2) The definition of symmetry constraints. This article defines plane of symmetry constraints in the center of symmetry to achieve the calculation effect of the $1 / 4$ which alternates whole model. After defining the effect shows in Fig. 4.
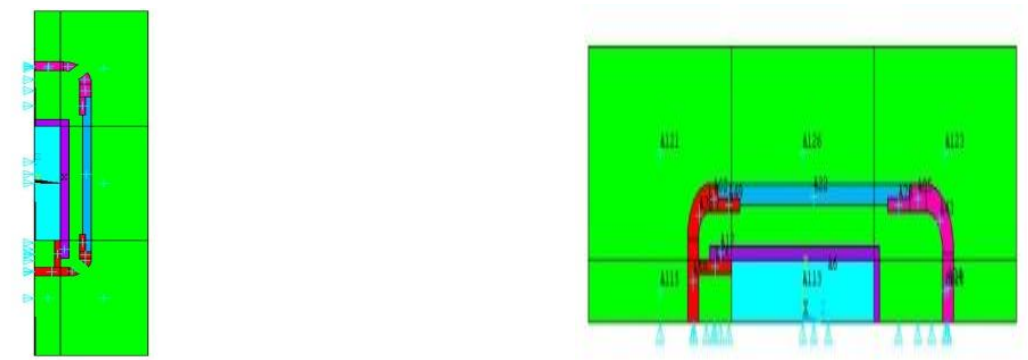

Figure 4. Zero displacement constraint (the left is the direction of the $\mathrm{x}$-axis and the right is $\mathrm{y}$-axis direction) 
(3)This article establishes a non-reflective constraint in the air outer boundary to achieve a borderless simulate of real situation, and defines solid-surface contact to simulate failure of the thread. The total time for solving set 0.0005 seconds, step of solving coefficient is 0.6. All results export in 0.000001 seconds interval[4].

(4) Exporting K file.

Parameters Selecting and Modifing. After exporting K file form, this article needs to add and modify keywords and parameters which Ansys can not be set:

(1) Make dynamite and unit into ALE unit[5].

(2) modifing material parameters. The four materials are defined pyrotechnics, ABS, air hardened aluminum alloy material and air material model.

(3) Adding ALE control keywords of CONTROL_ALE and fluid-solid coupling keyword of CONSTRAINED_LAGRANGE_IN_SOLID[6].

(4) Adding the initiation point to set keyword INITIAL_DETONATION, the initiation point is set at the center of the upper portion of the inner shell.

(5) Adding the erosion contact keywords CONTACT_ERODING_SURFACE between the inner shell and cover, middle housing and under cover is used for simulation on related collision and erosion[7].

After $\mathrm{K}$ file modification completed, this article saves, submits to Ansys Dyna solve to solve calculations.

\section{Analysis}

After solving, this article uses Ls-Prepost to open result file, after processing, this article analysises results.

Structural Analysis. After the fragments scattering, the effect with shell is divided into two parts, the first effect is between the circumferential portion and the housing fragment, as shown in 5 (a), the fragment is blocked by the middle shell, extrusion, deformation, slowing down and eventually retaining inside the housing. But the shell has only a very small elastic deformation and no damage. Fragments in the bottom of the inner shell fly toward the bottom with great acceleration in large explosive driven[8].

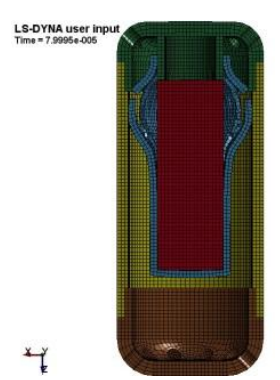

(a) $80 \mu \mathrm{s}$ inner shell fragments

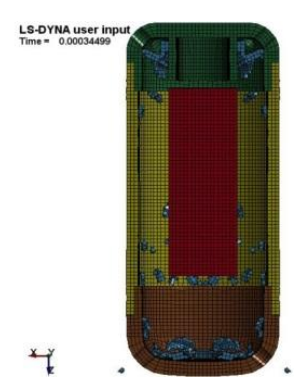

(b) $345 \mu$ s fragments scattering diagram

Figure 5. Fragment dispersion diagram

Its effect with the bottom cover is shown in Fig. 5 (b). Large fragments in the bottom of the inner shell most are blocked by the bottom cover, the bottom cover has small deformation and no bottom thread off, since the excessive vents lead to fragmentation flying, this has a chance, and the fragments are less than $0.01 \mathrm{~g}$, muzzle velocity is less than $150 \mathrm{~m} / \mathrm{s}$, the speed at $1 \mathrm{~m}$ attenuate to $100 \mathrm{~m} / \mathrm{s}$ or less, they belong to non-lethal fragments in the military standard and related literature, such fragments may be negligible. Therefore, we can draw fragments are completely blocked by shell and the shell has good condition.

Analysis of Overpressure We can obtain from the animation results that before $70 \mu \mathrm{s}$, overpressure generated by pyrotechnics is bound in ABS shell; after $70 \mu \mathrm{s}$, the inner shell cracks, the pressure enters 
from the inner shell into the outer shell; overpressure escapes gradually from the housing body orifices about $200 \mu \mathrm{s}$ which is in line with the explosion overpressure transport phenomena[9].

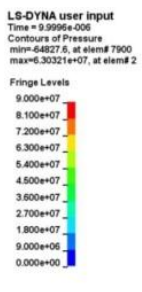

$\dddot{4}$

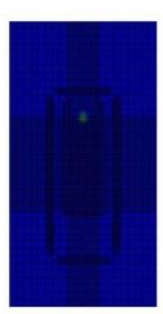

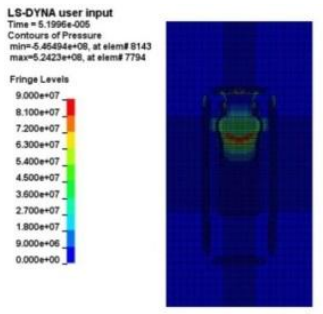

4

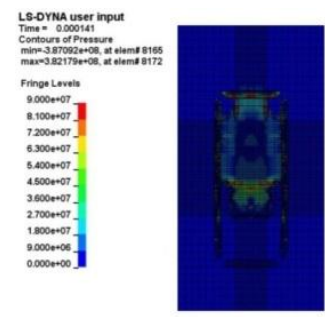

4.

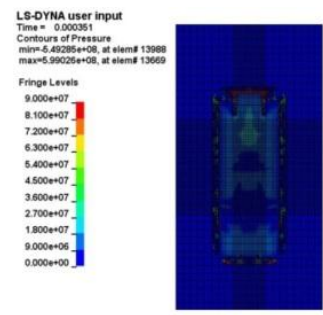

4

Figure 6. Overpressure cloud of elastomers in $10 \mu \mathrm{s}, 52 \mu \mathrm{s}, 141 \mu \mathrm{s}, 351 \mu \mathrm{s}$

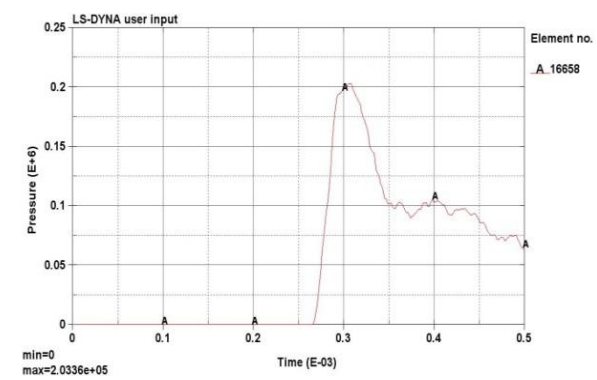

Figure 7. 16,658 units overpressure curve

This article takes air unit 16658 in the spout which is the largest overpressure, its overpressure curve is shown in Fig. 7, the peak overpressure is about $0.2 \mathrm{Mpa}$ which is in terms of peak SPL of $200 \mathrm{db}$, the upper and lower covers have a total of 20 vents, superimposed SPL is $213 \mathrm{db}$ which is reduced to $1.5 \mathrm{~m}$ at $137 \mathrm{db}$, it is close to the expected tactical and technical index.

\section{Experimental Verification}

In order to test the validity of the simulation results, we make bombs to structural reliability test.

This article selects open ground in the dirt floor and indoor and outdoor venues corner sequentially for tests, each site tests five ammunition. Fig. 8 is set to the ground pull release Fig. 9 is elastomer renderings after the explosion.

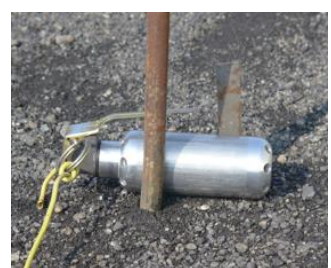

Figure 8. Plains pull release figure

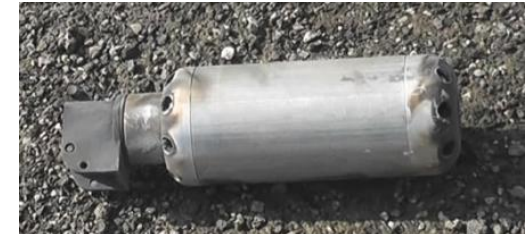

Figure 9 Elastomer renderings after the explosion

Result analysis:

(1) All ordnances explose normally, shell does not occur rupture, and the projectile fragments are stranded inside. The structure of the new bomb is feasible, outer shell does not deform substantially, it is consistent with the simulation results which verifies the correctness of the simulation and validity of the simulation parameters initially.

(2) the explosion of ammunition in the corner has a certain degree of displacement, but the displacement is small and there is no lasing, indicating part of the spout will not be blocked with lasing to cause destruction because new ball throw in the corner and other complex terrain[10].

This article measures weight changes before and after the explosion in the inner shell, the data is shown in Table 1: 
Table 1 Weight changes of the shell before and after the explosion

\begin{tabular}{|l|l|l|l|l|l|}
\hline weight before explosion $(\mathrm{g})$ & 7.20 & 7.20 & 7.19 & 7.18 & 7.18 \\
\hline weight after explosion $(\mathrm{g})$ & 6.84 & 6.82 & 6.83 & 6.66 & 6.72 \\
\hline weight changes $(\mathrm{g})$ & 0.36 & 0.38 & 0.36 & 0.52 & 0.46 \\
\hline
\end{tabular}

We can see fragments eject from the orifice at the maximum weight of 0.52g. Suppose it is a single fragment which flies hole at the highest speed $\mathrm{V}=198 \mathrm{~m} / \mathrm{s}$, then it has kinetic energy $10.2 \mathrm{~J}$, it has no personnel destruction.

\section{Conclusion}

This paper selects LS-DYNA as simulation software of the new glare blast bomb, and take simulation to theory design program of new light blast bomb. We can see from the results fragments is completely blocked by outer shell, the outer shell has good condition andno killing fragments splashing which is better. The connection between upper and lower lid does not fail, the inner shell and upper cover screw thread do not work because of the breakage inner shell, which is in line with actual needs, overpressure of vents is in line with tactical and technical index. The results show no fragmentation double structure completely eliminates the generation of anti-personnel fragmentation which achieves the design requirements. The structure is reasonably practicable, and the next step will be the further simulation and optimization to structural parameters.

\section{References}

[1] Zhao Shandong, Ma Yongzhong. Non-lethal weapons and police equipment [M]. Beijing: Weapon Industry Press, 2005, 12.Reference to a book:

[2] Mi Shuangshan, Liu Dongsheng, Xu Yaqing. Explosion simulation analysis based on flow solid coupling method [J]. Advanced manufacturing and management. 2008, 27 (03): 33-35.

[3] Zhang Qi, Zhang Ruojing. ALE method application in numerical simulation of the explosion [J]. Quarterly of Mechanics, 2005, 26 (4): 639-642.

[4] Gao Xuanneng, Liu Ying, Wang Shupeng. Blast wave pressure field analysis based on LS-DYNA of large space cylindrical shell [J]. Vibration and Shock. 2011, 30 (09): 70-75.

[5] Yao Wenyuan. Study on shock wave of the relief structure explosion [D]: [MS Thesis]. Chongqing: Civil Engineering, Chongqing University, 2011.

[6] John D.Reid. LS-DYNA EXAMPLES MANUAL [M]. USA: LIVERMORE SOFTWARE TECHNOLOGY CORPORATION, 1998.

[7] Ficket W, Davis W C. Detonation properties of condensed explosives calculated with equation of state based on intermolecular potentials [M], USA: LA-2712, 1962.

[8] Nicole D. Harasts, William Chung-Leung Chow, Mark Motyka. FLASH-BANG GRENADE WITH GREATER FLASH INTENSITY: USA, 8161883 [P]. 2012-08-24.

[9] Suri Bala. Contact Modeling in LS-DYNA- Some Recommendations-Part 1[M]. USA: LSTC, 2001.

[10]Livermore Software Technology Corpration. LS-DYNA Keyword User,s Manual(Version 971)[M/OL].2007-09-05.http://www.dynamore.de/documents/manuals/ls-dyna-manuals/ls-dyna -971-keyword. 